\title{
Age-Related Decrease of the Retinal Vasculature Area Identified with a Novel Computer-Aided Analysis System
}

\author{
Yuuka Yamamoto, ${ }^{1}$ Yoichiro Yamamoto, ${ }^{2,3}$ Atsushi Marugame, ${ }^{4}$ Maki Ogura, ${ }^{4}$ \\ Akira Saito, ${ }^{4}$ Kouichi Ohta, ${ }^{5}$ Manabu Fukumoto ${ }^{6}$ and Toshinori Murata ${ }^{1}$ \\ ${ }^{1}$ Department of Ophthalmology, Shinshu University School of Medicine, Matsumoto, Nagano, Japan \\ ${ }^{2}$ Division of Diagnostic Pathology, Nippon Medical School Hospital, Tokyo, Japan \\ ${ }^{3}$ Department of Molecular Medicine, Mayo Clinic Rochester, Rochester, MN, USA \\ ${ }^{4}$ Medical Solutions Division, NEC Corporation, Tokyo, Japan \\ ${ }^{5}$ Department of Ophthalmology, Matsumoto Dental University, Shiojiri, Nagano, Japan \\ ${ }^{6}$ Department of Pathology, Institute of Development, Aging and Cancer, Tohoku University, Sendai, Miyagi, Japan
}

Fundus photographs enable non-invasive analysis of the status of the microcirculation by directly observing the retinal vasculature. Retinal microvascular abnormalities are important clinical markers of hypertension and arteriosclerosis, but retinal microvascular changes can be observed in older individuals without hypertension. In this study, our goal is to elucidate the effects of aging on fundus vessels in the retinal photograph. We analyzed retinal vessels of 161 healthy volunteers (49.5 \pm 18.7 years, range 18-87) using in-house computer-aided measurement system to measure areas and diameters of all retinal vessels across the entire area of a retinal photograph. The vessels were segmented according to color, and then their area, size, length and thickness were measured by image processing. We also analyzed the correlation between total blood vessel area, age and mean arterial blood pressure (MABP). The decrease in total blood vessel area was dependent on both age and MABP. Moreover, decrease in blood vessel area was also correlated with age for the normotensive group. Furthermore, the slope of the regression line for retinal vessel area with MABP was significantly higher in participants aged $\leq 60$ years than in those aged over 60 years. Changes in retinal vessel area with aging were observed in both arterioles and venules. In conclusion, we found the significant decrease in retinal vessel area that is correlated well with calendar age. Therefore, we need to carefully apply traditional classifications of fundus examination for hypertensive retinopathy in older individuals.

Keywords: age; arteriosclerosis; computer-aided diagnosis; hypertension; retinal microvasculature.

Tohoku J. Exp. Med., 2012 Nov, 228 (3), 229-237. C 2012 Tohoku University Medical Press

Fundus photographs enable non-invasive analysis of the status of the microcirculation by directly observing the retinal vasculature. Retinal microvascular abnormalities such as generalized and focal arteriolar narrowing, arteriovenous nicking, and retinopathy (changes in the light reflex of the arteriole, microaneurysms, hemorrhages, and hard exudates) have been classified and investigated (Wagener et al. 1947; Scheie 1953; Leishman 1957; Stokoe and Turner 1966; Klein et al. 1993; Yu et al. 1998). These abnormalities are important clinical markers of hypertension and arteriosclerosis. However, it is difficult even for highly experienced ophthalmologists to detect and evaluate quantitative microvascular changes with reproducibility (Kagan et al. 1966).

Recent advances in computer-aided diagnosis (CAD) have made it possible to apply quantitative approaches for both analysis and diagnosis (Hubbard et al. 1999; Couper et al. 2002). CAD has been developed over the last 20 years following advances in computer technology and software algorithms as well as improved image capture techniques with higher resolution. Using CAD, the relationship between changes in retinal microvasculature and hypertension can be elucidated. Furthermore, cardiovascular diseases and stroke can be predicted by precisely measuring the diameter of the central retinal artery and vein (Hubbard et al. 1999; Kwa et al. 2002; Wong 2004; Wong and McIntosh 2005; Wong et al. 2006). The Atherosclerosis Risk in Communities study (ARIC) is the most widely accepted program for analyzing retinal photographs. However retinal microvascular changes can be observed in older individuals without hypertension (Leung et al. 2003; Wang et al. 2003; Wong 2004), these results could not be representative of overall changes in retinal vessels. As a result, there is often a divergence in assessment between

Received July 12, 2012; accepted September 20, 2012. Published online October 18, 2012; doi: 10.1620/tjem.228.229.

Correspondence: Manabu Fukumoto, M.D., Ph.D., Department of Pathology, Institute of Development, Aging and Cancer, Tohoku

University, 4-1 Seiryo-machi, Aoba-ku, Sendai, Miyagi 980-8575, Japan.

e-mail: fukumoto@idac.tohoku.ac.jp 
CAD and ophthalmologists. In this study, we measured the areas and diameters of all blood vessels in the retinal photograph using our in-house developed computer-aided analysis system in order to elucidate the effects of aging on fundus vessels.

\section{Materials and Methods}

\section{Study Population}

The study participants were volunteers at Matsumoto Dental University Hospital, Shiojiri, Japan. A total of 249 participants aged 18 to 87 years $(53.2 \pm 19.8$ years) (mean \pm S.D. $)$, were enrolled in this study.

\section{Ethics}

This study was performed according to the Declaration of Helsinki and was approved by the ethical committee, Faculty of Medicine, Shinshu University, Tohoku University and Matsumoto Dental University, Japan. We obtained informed consent from all participants involved in our study.

\section{Retinal Photography}

Color stereoscopic retinal photographs in the range of $45^{\circ}$ centered on the macula were taken for one or both eyes (only 1 field) using a fundus camera (model TRC-NW7SF, Topcon Optical Co., Tokyo, Japan). The format was JPEG and the dimension was $2,048 \times$ 1,536 pixels. Two or more ophthalmologists independently examined the photographs. Individuals with ophthalmoscopic abnormalities such as retinal hemorrhage, exudates, microaneurysms, advanced cataract and chorioretinal atrophies were excluded from this study.

\section{Measurement of Retinal Vessels}

All areas and diameters of retinal vessels that could be recognized by our measurement system were measured in pixels on digitized retinal photographs. For analyzing the retinal vasculature in digital images, vessels were first segmented according to color, and then their area, size, length and thickness were measured by image processing. Various algorithms for vessel extraction have been developed (Hoover et al. 2000). The quality of retinal images is strongly influenced by light intensity and conditions during image capture and also the skill of the operator. Therefore, in order to increase the overall quality and reduce the variation in quality, we developed an algorithm using multiple color channels, the green channel of RGB (Red, Green, and Blue) color system and the Hue value of HSV (Hue, Saturation, and Value) color system. In our algorithm, the complementary use of multiple color channels reduces the negative influence of area brightness on image quality. This technique stabilized the quality of the extracted import data even in a database which included low quality images (Fig. 1). The vasculature extracted from a color image was obtained as a binary image where the vasculature was white and non-vascularized regions were black (Fig. 2A). Thinning processes using the Hilditch thinning algorithm detected the center line of vessels in the original pixels (Fig. 2B). Distance transforma-
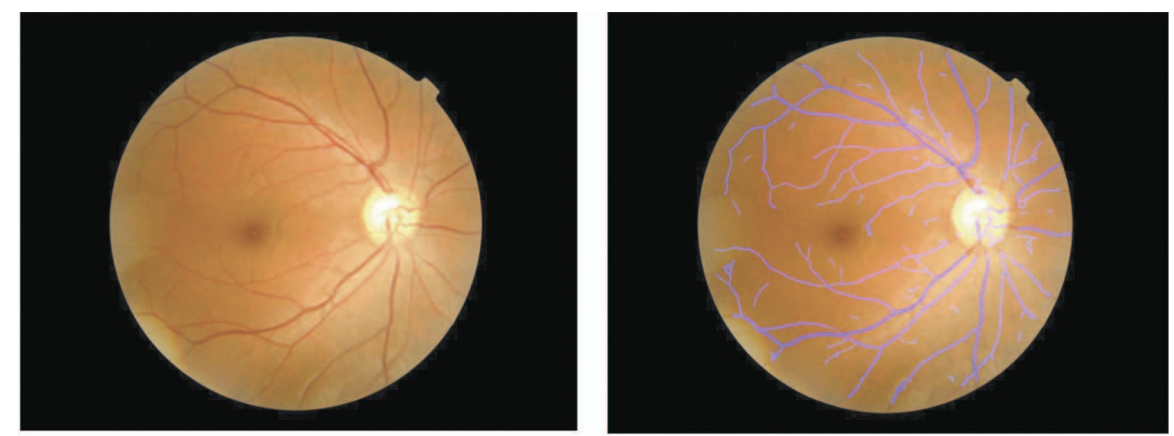

Normal

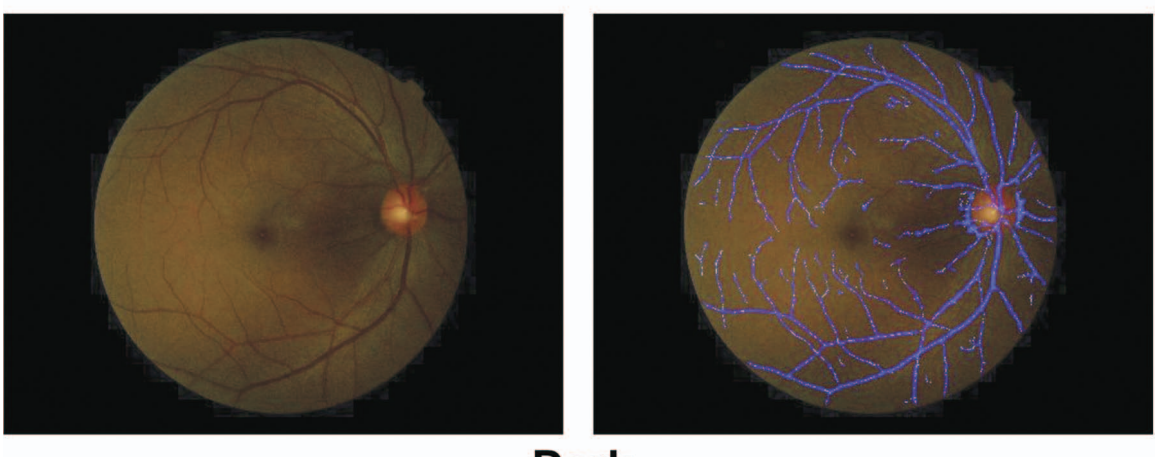

Dark

Fig. 1. Extraction of retinal vessels using our computer-aided measurement system.

For analyzing retinal vessels in digital photographs, vessels were first segmented with color information (blue color). The total blood vessel area in retinal images was calculated using factors such as area size, length, and thickness of retinal vessels extracted by image processing. We have developed an algorithm using multiple color channels, which can stabilize extraction qualities. The precision of the extraction is not different between photographs in normal quality (upper photo) and in low quality (lower photo). 

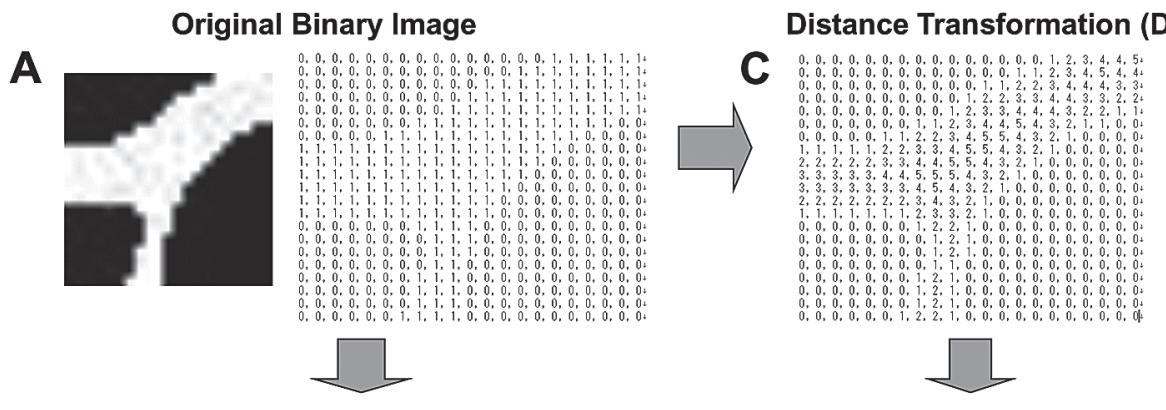

Hilditch Thinning
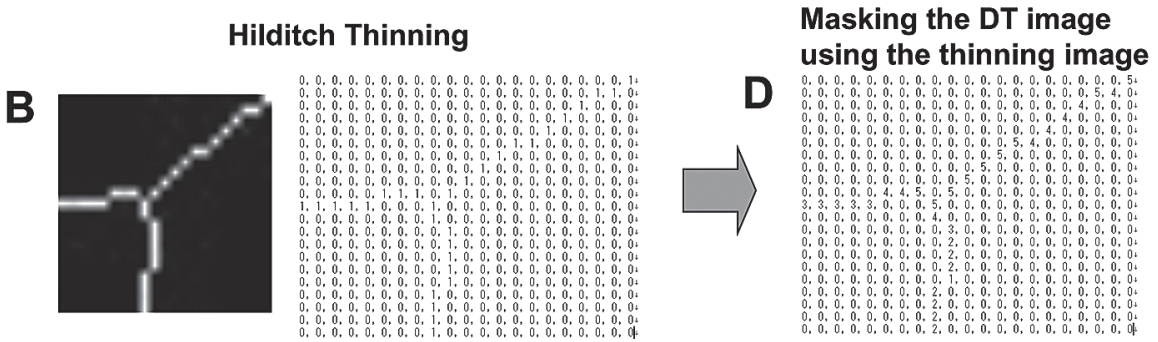

Fig. 2. The process of our computer-aided measurement methods.

A: Vessels extracted from a color image is transformed into a binary image where the vessel is in white and the background region is in black.

B: Hilditch thinning algorithm detects the center line of vessels in the original image.

$\mathrm{C}$ : The distance transformation process gives each pixel its distance value from boundaries between the vessel region (white) and the background region (black).

D: After a thinning image is overlaid on a distance transformed image, the calculated distance from the boundary is presented on the pixels of the center line.

tion processes assigned each pixel in white (vasculature) regions its distance value from boundaries between white regions and black (background, non-vascularized) regions (Fig. 2C). After a thinning image was overlaid on a distance transformed image, pixels on center lines were given a distance value from a boundary (Fig. 2D). Calculating strict vessel diameter to all center line pixels is very timeconsuming. The distance value of thinning pixels is a good approximation to a half of a vessel diameter (Fig. 3). Although the two values are not strictly equal where the change of a vessel diameter is striking, center lines and distance values almost matched by our method with very quickness. Therefore, we confirmed distance values on center lines as half that of vessel diameters. Total blood vessel area size can be computed from the sum of all distance values on center lines. Abnormally large distance values caused by false vessel extraction were removed in calculating a total blood vessel area size.

The retinal area measured was not significantly different between right and left eyes of the same individuals, therefore, we analyzed either the left or the right eye from each individual rather than both, enabling more resources to be used per analysis, resulting in higher quality results.

\section{Measurement of Blood Pressure}

Blood pressure was measured using an automated sphygmomanometer (model BP-203RVIII, Omron Healthcare Co., Kyoto, Japan) after subjects were comfortably seated for $5 \mathrm{~min}$. mean arterial blood pressure (MABP) was calculated as 0.33 of systolic blood pressure (SBP) plus 0.67 of diastolic blood pressure (DBP). Participants with $\mathrm{SBP} \geq 140 \mathrm{mmHg}$ or a $\mathrm{DBP} \geq 90 \mathrm{mmHg}$ were categorized in the hypertensive group.
Statistical Analysis

The distribution of the data was examined first by Shapiro-Wilk tests. Data were statistically analyzed, and the difference was considered statistically significant in case $p<0.05$. Pearson correlation coefficients $(r)$ were used to measure the relationship between age, total area of vessels and blood pressure. The difference between the regression slopes of the two groups was statistically examined, which employed $t$-test.

\section{Results}

The participants included 249 volunteers aged 18 to 87 years $(53.2 \pm 19.8$ years). We excluded cases with ophthalmoscopic abnormality and those of young participants who had difficulty with the analysis using the computer program because of excessive reflection of light on the posterior vitreous membrane. Retinal vasculature of 161 participants (49.5 \pm 18.7 years, range $18-87,56$ men and 105 women) was examined using our computer-aided measurement system elaborated in this study (Table 1). All areas and diameters of retinal vessels that could be recognized by our measurement system were measured in pixels on digitized retinal photographs (Fig. 1). A scatter plot matrix shows that the total area of blood vessels decreased with age (Pearson correlation coefficient: $r=-0.63 ; p<0.0001$ ) and mean arterial blood pressure (MABP) $(r=-0.44 ; p<$ 0.0001) (Fig. 4). Fig. 5 shows a path analysis based on multiple linear regression analysis. A path analysis based on multiple linear regressions analysis showed that the decrease in total blood vessel area was more dependent on age (standard partial regression coefficient: $\mathrm{SPR}=-0.54$; 

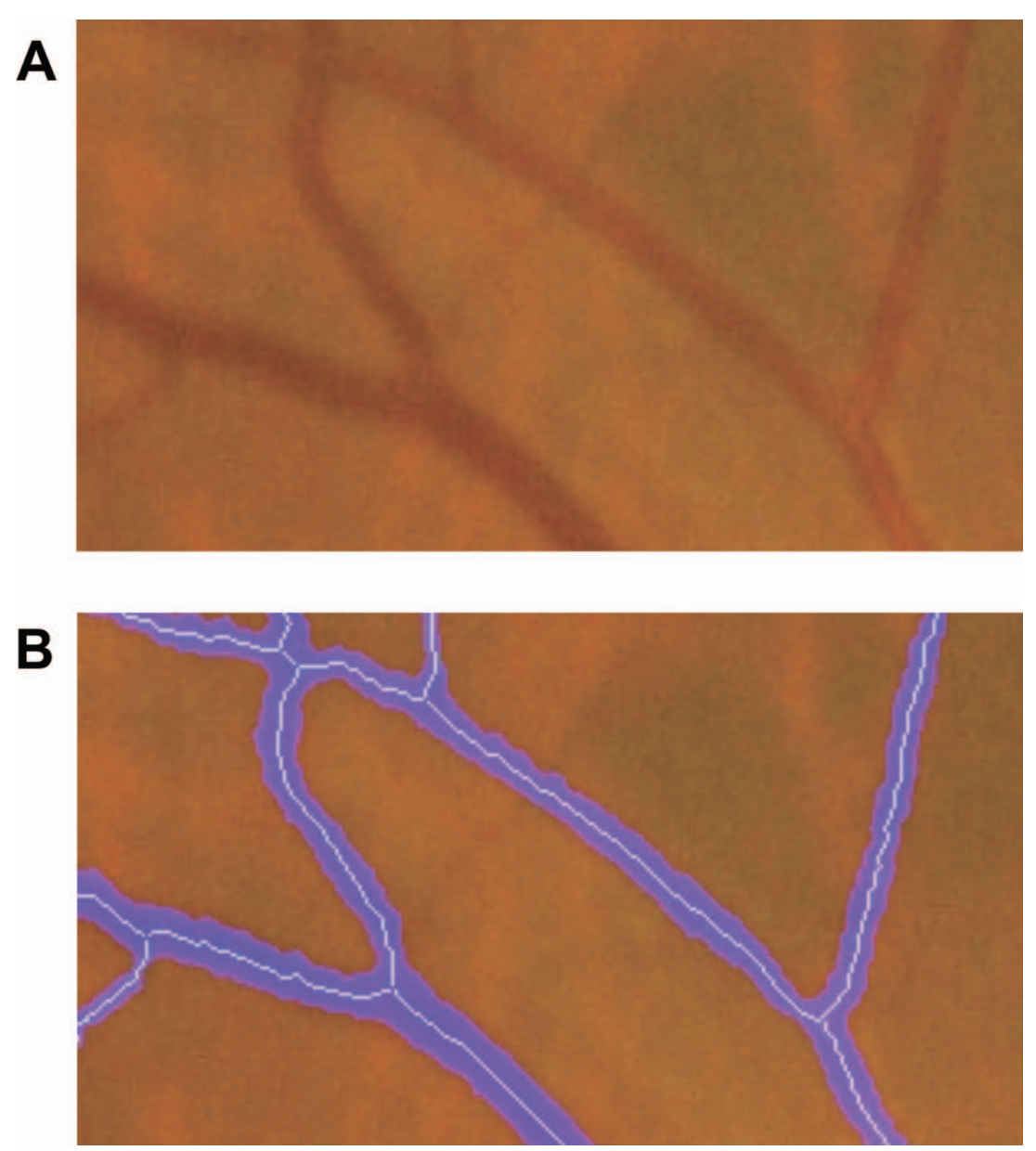

Fig. 3. The approximation of our computer-aided measurement methods.

A shows an original image of fundus vessels in the retinal photograph $(\times 400)$. B shows reconstructed vessel image after the thinning algorithm (blue area) overlapped onto the original image.

Table 1. Characteristics of all participants.

\begin{tabular}{lccc}
\hline & $\begin{array}{c}\text { All participants } \\
(n=161)\end{array}$ & $\begin{array}{c}\text { Male } \\
(n=56)\end{array}$ & \multicolumn{1}{c}{$\begin{array}{c}\text { Female } \\
(n=105)\end{array}$} \\
\hline Age (year) & $49.5 \pm 18.7$ & $57.4 \pm 14.6$ & $45.0 \pm 19.1$ \\
SBP (mmHg) & $124.2 \pm 18.0$ & $130.9 \pm 18.9$ & $120.6 \pm 16.5$ \\
DBP (mmHg) & $75.5 \pm 11.4$ & $81.3 \pm 11.0$ & $72.4 \pm 10.3$ \\
MABP (mmHg) & $91.5 \pm 13.1$ & $97.6 \pm 13.1$ & $88.3 \pm 11.9$ \\
\hline
\end{tabular}
pressure.

SBP, Systolic blood pressure; DBP, Diastolic blood pressure; MABP, Mean arterial blood

$p<0.001)$ than on MABP $(\mathrm{SPR}=-0.20 ; p<0.01)$. We also analyzed the correlation after excluding the hypertensive group ( $\mathrm{SBP} \geq 140 \mathrm{mmHg}$ or a $\mathrm{DBP} \geq 90 \mathrm{mmHg}$ ). In total, 128 subjects $(80 \%)$ remained and a strong inverse correlation between total blood vessel area and age still existed ( $r=-0.64$, Table 2, Fig. 6A). The inverse correlation was also observed in $72(45 \%)$ of participants even after excluding individuals with $\mathrm{SBP} \geq 120 \mathrm{mmHg}$ or DBP $\geq 80 \mathrm{mmHg}(r=-0.58 ; p<0.0001$, Table 3, Fig. 6B). In addition, the slope of the regression line for MABP was steeper in participants $\leq 60$ years $(r=-0.47 ; p<0.0001)$ than in those $>60$ years $(r=-0.16 ; p=0.25)$. The difference between the regression slopes of the two groups was significant ( $t$-test, $p<0.05)$ (Fig. 7).

Furthermore, we analyzed arterioles and venules separately in 27 cases; 9 of which were randomly selected from the following 3 groups: younger individuals (aged $<30$ years), middle-aged (aged 40-50 years), and older (aged $>60$ years) after excluding subjects with hypertension. Two ophthalmologists performed independent qualitative 


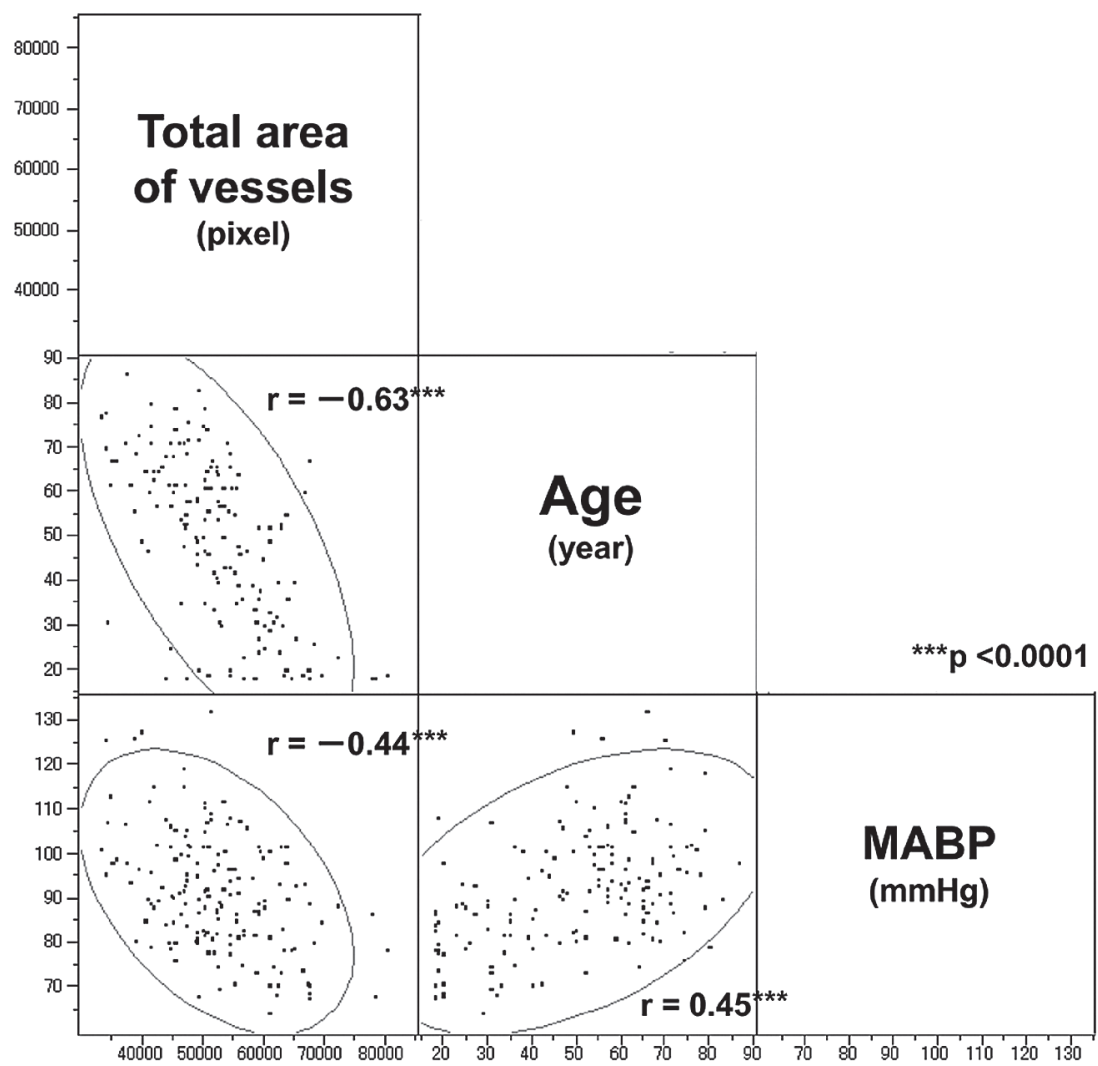

Fig. 4. Correlation of age, total blood vessel area and MABP.

Scatter plot matrix of the total blood vessel area and age and MABP with the $95 \%$ prediction confidence ellipses.

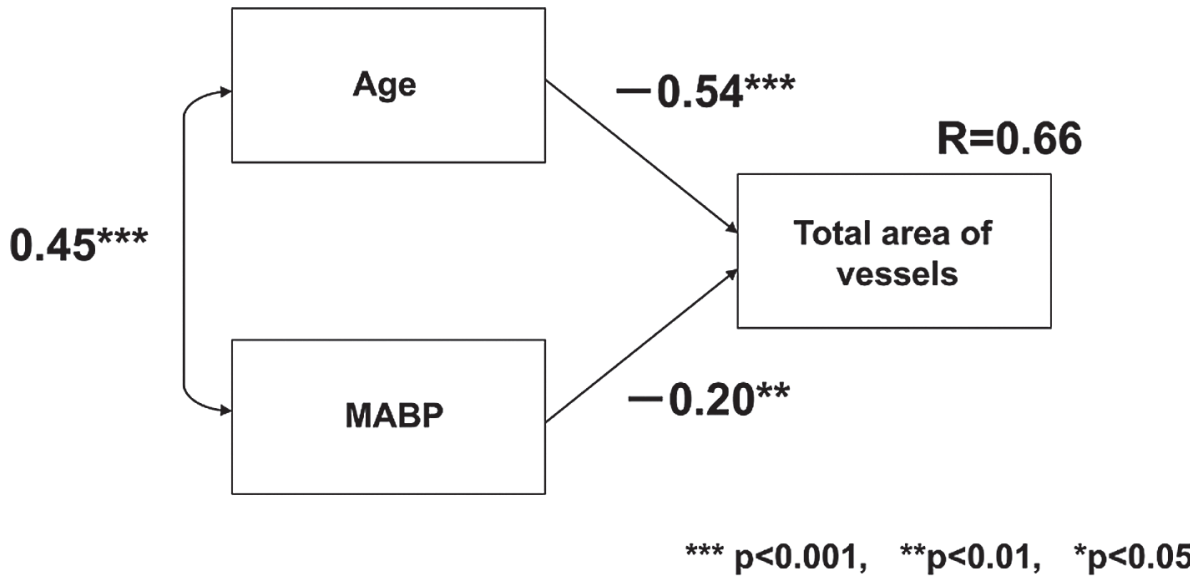

Fig. 5. Relevance of age and MABP to the total blood vessel area presented by the path analysis.

Standard partial regression coefficient (SPR) of the total blood vessel area on age is -0.54 . SPR of the total blood vessel area on MABP is -0.20 . Regression coefficient between age and MABP is 0.45 . Determination coefficient $(\mathrm{R})$ is 0.66 .

examination of blood vessels in the original color photographs in order to classify them into either arterioles or venules: where there was ambiguity in initial classification the ophthalmologists preformed re-examination in order to arrive at a consensus classification. This was required as the computer-aided measurement system could not discriminate between arterioles and venules. The total area of both arterioles $(r=-0.82 ; p<0.0001)$ and venules $(r=-0.78 ; p$
$<0.0001)$ was correlated with age (Fig. 8A). On the other hand, the correlation coefficient between MABP to the area was not significant $(r=-0.34 ; p=0.08$ and $r=-0.26$; $p=$ 0.19) (Fig. 8B).

\section{Discussion}

We analyzed retinal vessels of 161 healthy volunteers using computer-aided measurement system to measure 
Table 2. Characteristics of participants.

\begin{tabular}{lccc}
\hline & Hypertensive group & Non- hypertensive group & \\
& $\mathrm{SPB} \geq 140$ or DBP $\geq 90(n=34)$ & $\mathrm{SPB}<140$ and DBP $<90(n=128)$ & $<0.001$ \\
\hline Age (year) & $61.1 \pm 13.9$ & $46.4 \pm 18.6$ & $<0.001$ \\
SBP (mmHg) & $150.7 \pm 10.5$ & $117.4 \pm 12.3$ & $<0.001$ \\
DBP (mmHg) & $90.1 \pm 8.9$ & $71.8 \pm 8.7$ & $<0.001$ \\
MABP (mmHg) & $110.1 \pm 8.5$ & $86.8 \pm 9.4$ & \\
\hline
\end{tabular}

SBP, Systolic blood pressure; DBP, Diastolic blood pressure; MABP, Mean arterial blood pressure.

The participants was classified by blood pressure by two phases.

Table 3. Characteristics of participants.

\begin{tabular}{lccc}
\hline & Without normotensive group & Normotensive group & $p$ \\
& $\mathrm{SPB} \geq 120$ or DBP $\geq 80(n=90)$ & $\mathrm{SPB}<120$ and DBP $<80(n=72)$ & $<0.001$ \\
Age (year) & $57.0 \pm 14.7$ & $40.1 \pm 19.1$ & $<0.001$ \\
SBP (mmHg) & $137.1 \pm 13.2$ & $108.5 \pm 8.1$ & $<0.001$ \\
DBP (mmHg) & $82.7 \pm 9.2$ & $66.7 \pm 6.9$ & $<0.001$ \\
MABP (mmHg) & $100.7 \pm 9.8$ & $80.5 \pm 6.8$ & \\
\hline
\end{tabular}

SBP, Systolic blood pressure; DBP, Diastolic blood pressure; MABP, Mean arterial blood pressure.

The participants was classified by blood pressure by two phases.
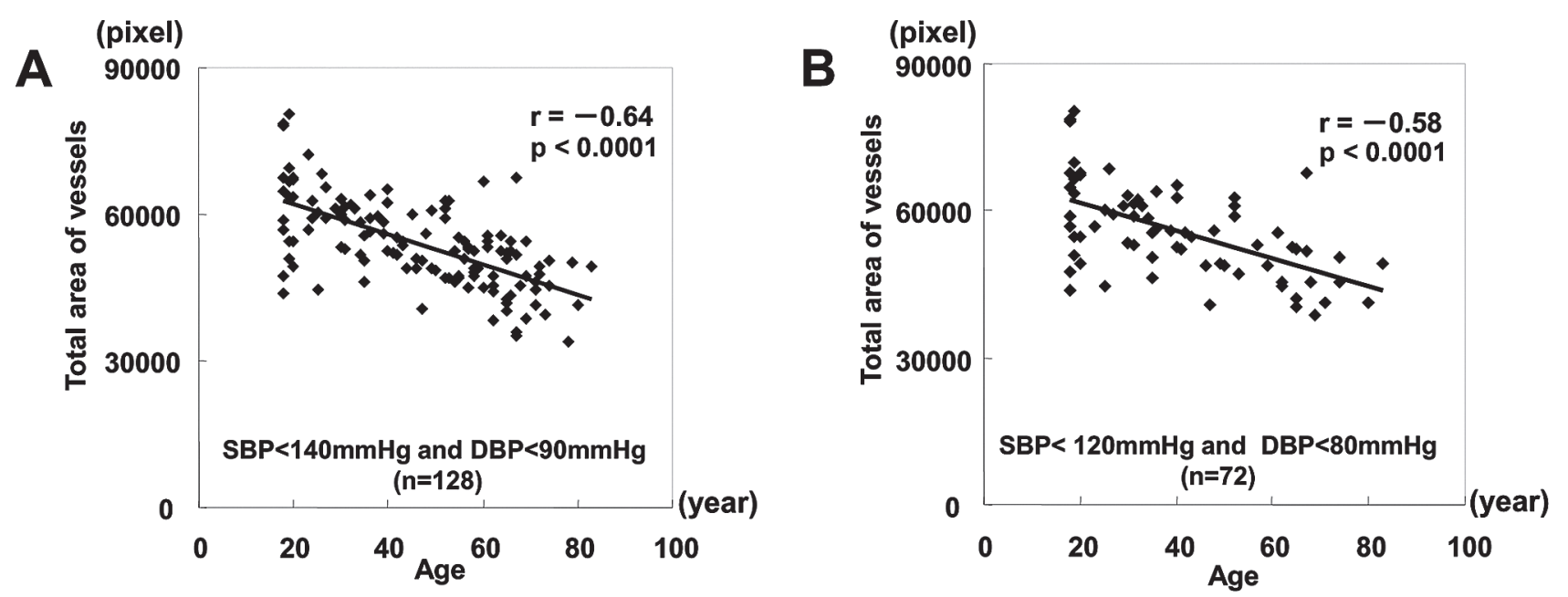

Fig. 6. Correlation between age and the total area of vessels in non-hypertensive group. Inverse correlation between age and total area of vessels both in group A (SBP $<140 \mathrm{mmHg}, \mathrm{DBP}<90 \mathrm{mmHg})$ and group $\mathrm{B}(\mathrm{SBP}<120 \mathrm{mmHg}$, DBP $<80 \mathrm{mmHg})$.

areas and diameters of all retinal vessels across the entire area of a retinal photograph. The decrease in total area of retinal vessels was strongly associated with age (Fig. 9). The inverse correlation between total vessel area and age was confirmed even in the normotensive group. In addition, the slope of the regression line of the total area of vessels for MABP was significantly higher in those aged $\leq 60$ years than in those aged $>60$ years. These are consistent with the recent report that fundus findings are a poor predictor of the risk of systemic disorders such as coronary artery diseases for those aged 75 years and older compared with middle aged individuals (Wong et al. 2003; van den
Born et al. 2005).

We independently analyzed the arterioles and venules of young, middle aged, and older participants in order to confirm the effect of blood pressure and aging on arterioles and venules. In our study, both arteriole and venule areas were correlated with age. Our method is simple to use and permits easy detection and area determination of all fundus vessels in a retinal photograph, but cannot discriminate between arterioles and venules. However our study indicated that the retinal areas of both arterioles and venules exhibit similar age-related patterns in for healthy volunteers of this study. 


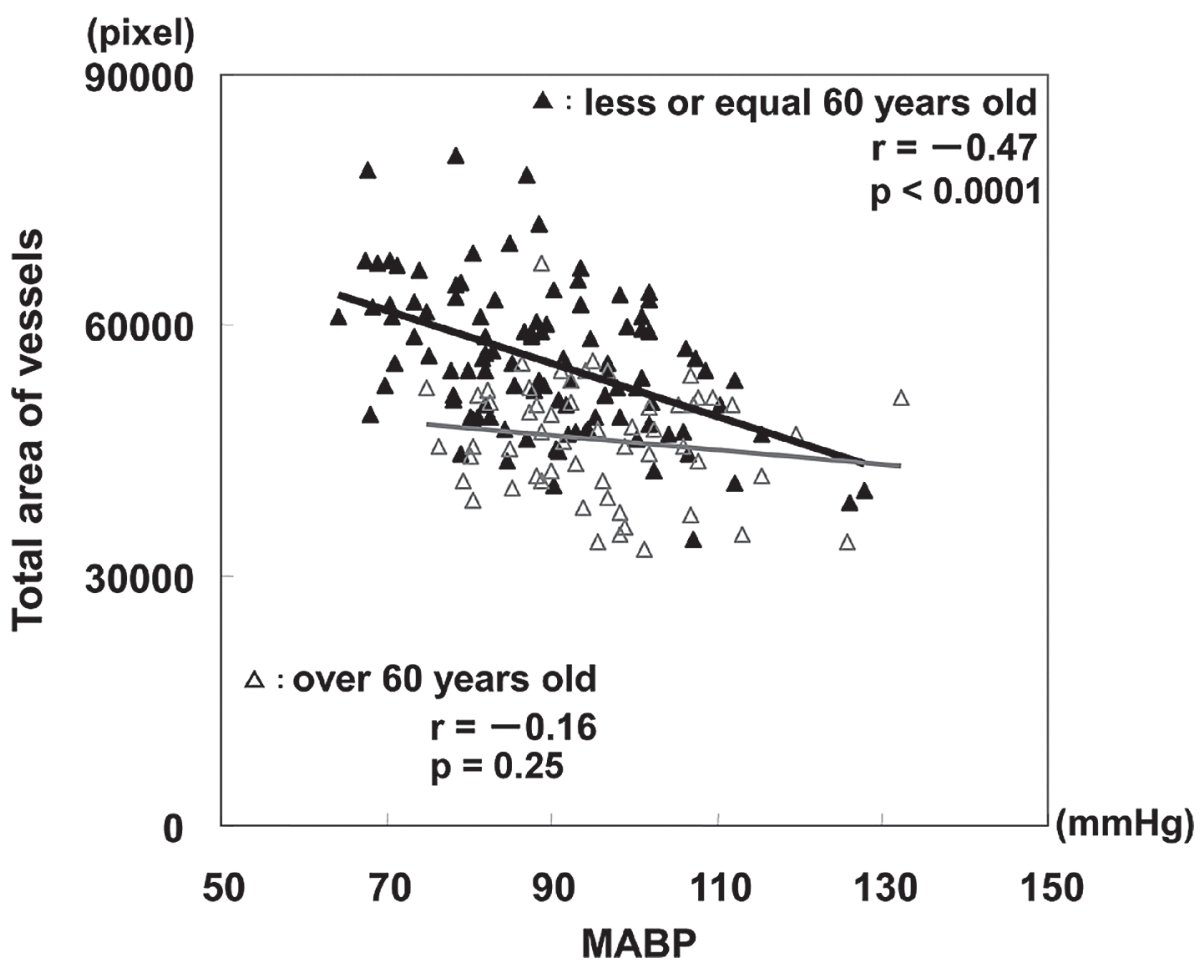

Fig.7. Correlation between MABP and the total area of blood vessels.

The total area of blood vessels was correlated with MABP in participants aged $\leq 60$ years $(r=-0.47)$, but not in those aged $>60$ years $(r=-0.16)$.
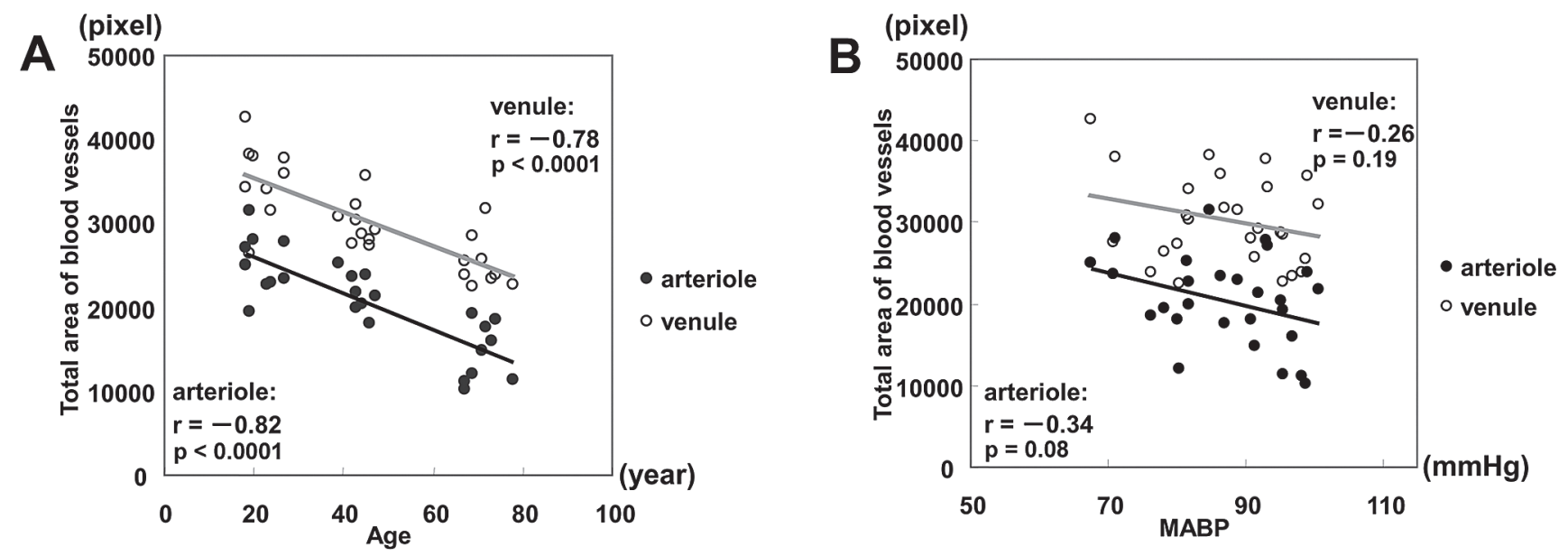

Fig. 8. Correlation between age and the total are of blood vessels, and MABP and the total area of blood vessels. Both the total arteriole area $(r=-0.82 ; p<0.0001)$ and venule area $(r=-0.78 ; p<0.0001)$ were correlated with age (A) but not with MABP (B).

Corneal curvature, refractive errors and axial length affect the magnification of retinal photographs. Several formulae to calculate their absolute values have been developed (Parr and Spears 1974; Bennett et al. 1994). Knudtson et al. reported a size correcting formula for axial length (Knudtson et al. 2003). In the present study, the longer the axial length was the narrower the retinal vessel diameter was in a photograph. Although we did not estimate axial length of the participants, it is known that axial length of older individuals is smaller in Japanese participants than younger individuals (Esaki et al. 2000; Hamano 2001). Therefore, the diameter of retinal vessels may be slightly overestimated in older participants and the evaluated retinal vessel area might be greater than the actual value.

Branch retinal vein occlusion (BRVO) is a frequent retinal vascular disease with an incidence of 2.14/1,000/ year in the population over 40 years of age (David et al. 1988). It may cause immediate vision loss due to reduced blood perfusion and subsequent retinal hypoxia (Kanski 2007). Correlations between BRVO and systemic disorder 

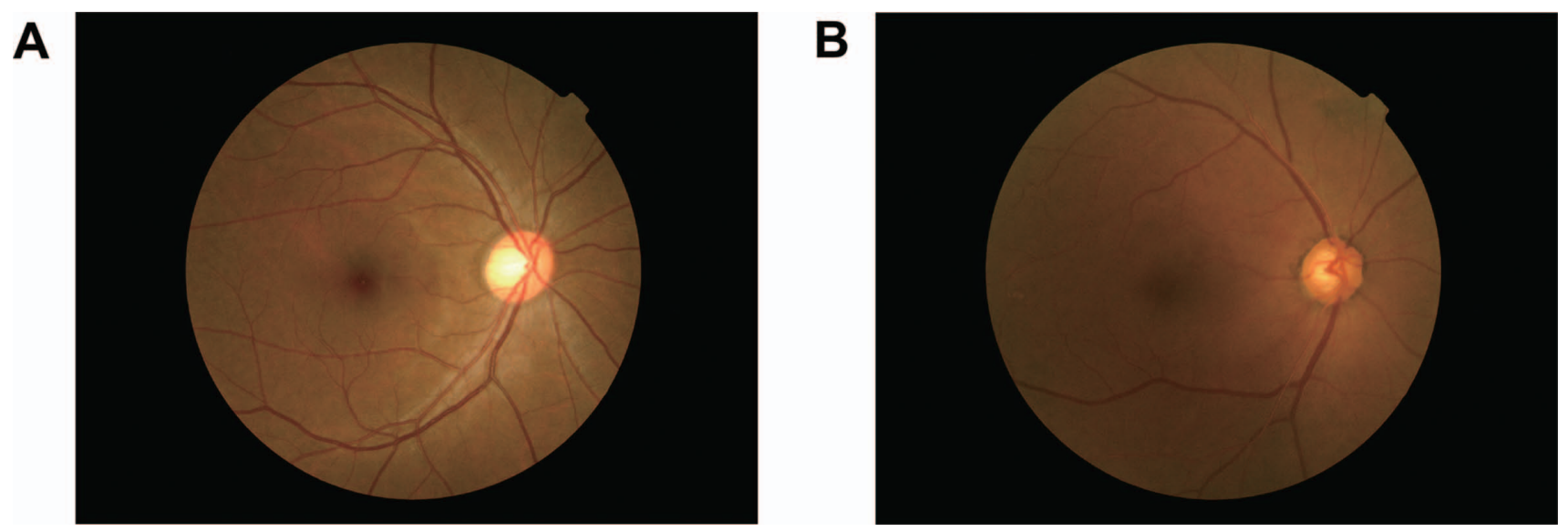

Fig. 9. Representative photographs from young and elderly subjects.

A: From a young subject ( 30 years) and B: From elderly ( 81 years).

Thin vessels can be more easily recognized in younger subjects than elder subjects.

such as hypertension or diabetes mellitus have been reported. However, the cause of BRVO in old people without these systemic disorders remains unclear (Klein et al. 2000; Hayreh et al. 2001). Our results suggest that the total blood vessel area decreases along with aging and that MABP has a small impact on people aged over 60 . We think that the age-related decrease of total blood vessel area on ocular fundus may be one of causes for BRVO.

In conclusion, we found that significant decreases in retinal vessel area correlated well with calendar age using our in-house developed computer-aided measurement system. A strong correlation was observed between age and retinal vessel area in normotensive participants. Our study warns that ophthalmologists might have overestimated retinal vessel changes due to hypertension when the fundus of older participants was examined. Ophthalmologists should carefully consider the age associated decrease of vessel area when evaluating retinal vessels in older individuals.

\section{Acknowledgments}

The authors thank Atsuko Sato, M.D., Emi Fukui, M.D., co-medical staff (Matsumoto Dental University Hospital) for management of participants.

\section{Specific Remarks}

This manuscript has been seen approved by all authors, and is not under consideration for publication elsewhere in similar form, in any language, except in abstract form.

\section{Conflict of Interest}

We have no conflicting interest affecting the present study.

\section{References}

Atherosclerosis Risk in Communities (ARIC) Study Research Group. ARIC Retinal Photography Protocol, Manual 14A, and ARIC Retinal Reading Center Protocol, Manual 14B. Chapel Hill, NC: ARIC Coordinating Center, Department of Biostatistics (CSCC), University of North Carolina, CB\#
8030, Suite 203, Nationbank Plaza, 137 E. Franklin St., Chapel Hill, NC 27514.

Bennett, A.G., Rudnicka, A.R. \& Edgar, D.F. (1994) Improvements on Littmann's method of determining the size of retinal features by fundus photography. Graefes Arch. Clin. Exp. Ophthalmol., 232, 361-367.

Couper, D.J., Klein, R., Hubbard, L.D., Wong, T.Y., Sorlie, P.D., Cooper, L.S., Brothers, R.J. \& Nieto, F.J. (2002) Reliability of retinal photography in the assessment of retinal microvascular characteristics: the Atherosclerosis Risk in Communities Study. Am. J. Ophthalmol., 133, 78-88.

David, R., Zangwill, L., Badarna, M. \& Yassur, Y. (1988) Epidemiology of retinal vein occlusion and its association with glaucoma and increased intraocular pressure. Ophthalmologica, 197, 69-74.

Esaki, K., Ishikawa, H., Liebmann, J.M., Greenfield, D.S., Uji, Y. \& Ritch, R. (2000) Angle recess area decreases with age in normal Japanese. Jpn. J. Ophthalmol., 44, 46-51.

Hamano, H. (2001) Comparison of Axial Length between Younger and Older Adult in Japan. Journal of the Eye, 18, 1555-1558.

Hayreh, S.S., Zimmerman, B., McCarthy, M.J. \& Podhajsky, P. (2001) Systemic diseases associated with various types of retinal vein occlusion. Am. J. Ophthalmol., 131, 61-77.

Hoover, A., Kouznetsova, V. \& Goldbaum, M. (2000) Locating blood vessels in retinal images by piecewise threshold probing of a matched filter response. IEEE Trans. Med. Imaging, 19, 203-210

Hubbard, L.D., Brothers, R.J., King, W.N., Clegg, L.X., Klein, R., Cooper, L.S., Sharrett, A.R., Davis, M.D. \& Cai, J., (1999) Methods for evaluation of retinal microvascular abnormalities associated with hypertension/sclerosis in the Atherosclerosis Risk in Communities Study. Ophthalmology, 106, 2269-2280.

Kagan, A., Aureli, E. \& Dobree, J. (1966) A note on signs in the fundus oculi and arterial hypertension: conventional assessment and significance. Bull. World Health Organ., 34, 955-960.

Kanski, J.J. (2007) Clinical ophthalmology: a systematic approach, 6th ed., Elsevier-Butterworth- Heinemann, Edingburgh. pp. 584-592.

Klein, R., Klein, B.E., Moss, S.E. \& Meuer, S.M. (2000) The epidemiology of retinal vein occlusion: the Beaver Dam Eye Study. Trans. Am. Ophthalmol. Soc., 98, 133-141.

Klein, R., Klein, B.E., Moss, S.E. \& Wang, Q. (1993) Blood pressure, hypertension and retinopathy in a population. Trans. Am. Ophthalmol. Soc., 91, 207-226. 
Knudtson, M.D., Lee, K.E., Hubbard, L.D., Wong, T.Y., Klein, R. \& Klein, B.E. (2003) Revised formulas for summarizing retinal vessel diameters. Curr. Eye Res., 27, 143-149.

Kwa, V.I., van der Sande, J.J., Stam, J., Tijmes, N. \& Vrooland, J.L. (2002) Retinal arterial changes correlate with cerebral smallvessel disease. Neurology, 59, 1536-1540.

Leishman, R. (1957) The eye in general vascular disease: hypertension and arteriosclerosis. Br. J. Ophthalmol., 41, 641-701.

Leung, H., Wang, J.J., Rochtchina, E., Tan, A.G., Wong, T.Y., Klein, R., Hubbard, L.D. \& Mitchell, P. (2003) Relationships between age, blood pressure, and retinal vessel diameters in an older population. Invest. Ophthalmol. Vis. Sci., 44, 29002904.

Parr, J.C. \& Spears, G.F. (1974) Mathematic relationships between the width of a retinal artery and the widths of its branches. Am. J. Ophthalmol., 77, 478-483.

Scheie, H.G. (1953) Evaluation of ophthalmoscopic changes of hypertension and arteriolar sclerosis. AMA Arch. Ophthalmol., 49, 117-138.

Stokoe, N.L. \& Turner, R.W. (1966) Normal retinal vascular pattern. Arteriovenous ratio as a measure of arterial calibre. Br. J. Ophthalmol., 50, 21-40.

van den Born, B.J., Hulsman, C.A., Hoekstra, J.B., Schlingemann, R.O. \& van Montfrans, G.A. (2005) Value of routine funduscopy in patients with hypertension: systematic review. $B M J$, 331, 73.

Wagener, H.P., Clay, G.E. \& Gipner, J.F. (1947) Classification of
Retinal Lesions in the Presence of Vascular Hypertension. Report submitted to the American Ophthalmological Society by the committee on Classification of Hypertensive Disease of the Retina. Trans. Am. Ophthalmol. Soc., 45, 57-73.

Wang, J.J., Mitchell, P., Leung, H., Rochtchina, E., Wong, T.Y. \& Klein, R. (2003) Hypertensive retinal vessel wall signs in a general older population: the Blue Mountains Eye Study. Hypertension, 42, 534-541.

Wong, T.Y. (2004) Is retinal photography useful in the measurement of stroke risk? Lancet Neurol., 3, 179-183.

Wong, T.Y., Kamineni, A., Klein, R., Sharrett, A.R., Klein, B.E., Siscovick, D.S., Cushman, M. \& Duncan, B.B. (2006) Quantitative retinal venular caliber and risk of cardiovascular disease in older persons: the cardiovascular health study. Arch. Intern. Med., 166, 2388-2394.

Wong, T.Y., Klein, R., Nieto, F.J., Klein, B.E., Sharrett, A.R., Meuer, S.M., Hubbard, L.D. \& Tielsch, J.M. (2003) Retinal microvascular abnormalities and 10-year cardiovascular mortality: a population-based case-control study. Ophthalmology, 110, 933-940.

Wong, T.Y. \& McIntosh, R. (2005) Hypertensive retinopathy signs as risk indicators of cardiovascular morbidity and mortality. Br. Med. Bull., 73, 57-70.

Yu, T., Mitchell, P., Berry, G., Li, W. \& Wang, J.J. (1998) Retinopathy in older persons without diabetes and its relationship to hypertension. Arch. Ophthalmol., 116, 83-89. 